\title{
Kausalkonstruktionen mit Adjektiv im freien und fixierten Gebrauch
}

\author{
Sabine De Knop (Brüssel) und Fabio Mollica (Mailand)*
}

\begin{abstract}
The study describes the semantic and conceptual variation of causal constructions with an adjective as they are realized in German, French and Italian. In the framework of Goldberg's (cf. 1995 and 2006) Construction Grammar, it discusses some defining properties of the analyzed constructions, like the formal and semantic (non-)predictability and their possible phraseological status based on metonymy or metaphor. Several constructions can be identified which convey different meanings, i. e. causality and/or excessiveness. The instantiations of the causal construction are linked to each other by inheritance links which make it possible to describe the variation as a network of constructions. The description of the examples' variation with their defining properties allows us to advocate a continuum between free and fixed instantiations of the constructions and accordingly between Construction Grammar and Phraseology.
\end{abstract}

\section{$1 \quad$ Einleitung}

Der vorliegende Beitrag untersucht kausale Strukturen mit einem Adjektiv (ADJ) gefolgt von einer Präpositionalphrase (PP), und zwar in den Sprachen Deutsch, Französisch und Italienisch. Zur Veranschaulichung können folgende Beispiele dienen: ${ }^{1}$

(1) dt. Ihre Hände waren blau vor Kälte.

(2) fr. La jeune fille était devenue rouge de honte.

Die junge Frau war geworden rot von Scham

,Die junge Frau war vor Scham rot geworden.

(3) it. Sono rosso di rabbia.

Bin rot von Wut

,Ich bin rot vor Wut.

\footnotetext{
* Der vorliegende Artikel ist das Ergebnis der gemeinsamen Diskussion; Sabine De Knop hat die Punkte 1-2.3, Fabio Mollica die Punkte 2.4-4 verfasst. An dieser Stelle möchten wir uns bei Carmen Mellado Blanco, Elmar Schafroth, Alexander Ziem und bei einem anonymen Gutachter für hilfreiche Anregungen und Kommentare zu einer früheren Fassung bedanken.

${ }^{1}$ Die Beispiele aus dem Französischen und Italienischen werden nach dem Leipziger Glossenmodell übersetzt, und zwar nach Regel 2, ,morpheme-to-morpheme correspondence“, siehe etwa https://www.eva.mpg.de/lingua/ resources/glossing-rules.php.
}

Linguistik online 90, 3/18 - http://dx.doi.org/10.13092/lo.90.4317

CC by 3.0 
(4) it. Natalie sta morendo di sete.

Natalie ist sterbend von Durst

,Natalie stirbt gerade vor Durst.

(5) fr. Jean est fou d'amour.

Jean ist verrückt von Liebe

,Jean ist verrückt vor Liebe.

Die mit den Beispielen (1)-(5) vermittelte Bedeutung ist primär die einer Kausalität, d. h. einer Ursache-Wirkung-Relation, die mit der Präpositionalphrase (Ursache) und dem Adjektiv (Wirkung, Effekt) zum Ausdruck gebracht wird. Die Kookkurrenz des Adjektivs und der Präpositionalphrase in ihrer kausalen Lesart wird im Folgenden [ADJ+PP] kausal schematisch dargestellt. Obwohl diese Beispiele alle dieselbe syntaktische Struktur aufweisen, sind sie durch eine semantische Variation gekennzeichnet. Bei näherer Betrachtung sind verschiedene Bedeutungslesarten zu erkennen. In den ersten Beispielen tritt ein Farbadjektiv auf, und es ist die tatsächliche Farbe des Körperteils (Hände in (1) oder Gesicht in (2)) als Stelle für die Veränderung gemeint. In Beispiel (3) wird auch ein Farbadjektiv gebraucht, allerdings wird eher ein extremer Zustand ausgedrückt - zumindest wenn es nicht wörtlich verstanden wird (siehe Abschnitt 2.3). Die Beispiele (4) und (5) vermitteln auch einen extremen Zustand, etwas Exzessives, allerdings nicht mit einem Farbadjektiv, sondern mit dem Sprachzeichen mort (,tot') oder fou (,verrückt').

Weiter unterscheiden sich die fünf Beispiele durch ihren Lexikalisierungsgrad. In (4) und (5) bringen die Adjektive keine wortwörtliche Bedeutung zum Ausdruck, sondern eher eine übertragene, metaphorische: Der Experiencer (ausgedrückt durch das Subjekt) ist nicht tatsächlich verrückt (fou in Beispiel (5)) oder wirklich am Sterben (sta morendo in Beispiel (4)). Diese Belege gehören zu den phraseologischen Ausdrucksmöglichkeiten der untersuchten Sprachen (zu Beispiel (3) cf. 2.3).

Die besprochenen Beispiele entsprechen Konstruktionen im Sinne von Goldberg $(1995,2006)$, d. h. Form-Bedeutungspaaren, die „form- und inhaltsbezogene Informationseinheiten bündel[n] und konventionell miteinander verbinde[n]“ (Ziem/Lasch 2013: 14). In ihrem ersten Buch (1995) plädierte Goldberg für das Argument der Nichtprädiktabilität als definitorische Bedingung für Konstruktionen. Nichtprädiktabel sind die Beispiele (1)-(5) sowohl in ihrer Form als auch in ihrer Bedeutung. Formal kann man die aktualisierten Präpositionen nicht durch andere Präpositionen mit kausaler Bedeutung ersetzen. So kann bei (1) vor nicht durch von ersetzt werden. Auf dieselbe Weise sind auch die Präpositionen it. di und frz. de nicht austauschbar. Semantische Nichtprädiktabilität sieht vor, dass sich die Gesamtbedeutung einer Konstruktion nicht einfach kompositionell aus der Bedeutung der einzelnen konstituierenden Bestandteile ergibt. Dies ist zu beobachten in den Beispielen (3)-(5): Ich bin nicht wirklich rot (3), Natalie ist nicht wirklich am Sterben (4) und Jean ist nicht tatsächlich verrückt (5). Die Bedeutung der ersten Belege (1) und (2) dagegen ist kompositionell zu verstehen; trotzdem sind sie auch als Konstruktionen aufzufassen, da ihre Struktur in den untersuchten Sprachen stark verfestigt (Englisch entrenched) und mit einer gewissen Frequenz vertreten ist. Diese Konstruktionsauffassung entspricht Goldbergs späterer erweiterter Definition 
(2006). Wir werden in Abschnitt 2.3. näher auf diese Eigenschaften eingehen. Im Folgenden gehen wir von dieser erweiterten Definition aus.

Goldbergs (2006) erweiterte Konstruktionsauffassung bietet neue Wege, um mehr oder weniger lexikalisierte oder idiomatisierte Strukturen genauer zu differenzieren und einheitlicher zu beschreiben. Verschiedene Verfestigungsgrade können unterschieden werden, die dank dem Konzept des Konstruktionsnetzwerks zu erfassen sind. Solch ein Netzwerk beschreibt verwandte Strukturen als ein organisiertes Inventar an Konstruktionen. Dabei werden folgende Fragen interessant: (i) Sind die besprochenen Beispiele Instanziierungen derselben Konstruktion oder entsprechen sie vielleicht verschiedenen Konstruktionen? (ii) Wie lassen sich die wörtlichen Kausalkonstruktionen von den phraseologischen unterscheiden? (iii) Wie lässt sich die Grenze zwischen einer wörtlichen Konstruktion, einem Phraseologismus und einer so genannten Phrasem-Konstruktion (im Sinne von Dobrovol'skij 2011) wie in Beispiel (4) definieren? (iv) Inwiefern bietet die Konstruktionsgrammatik ein effizientes Modell zur Analyse und Beschreibung phraseologischer Konstruktionen? (v) Welche Erkenntnisse bringt eine kontrastive Analyse (Deutsch-Französisch-Italienisch) solcher Konstruktionen? Ziel der Untersuchung ist es, eine Antwort auf diese Fragen zu bringen und zu zeigen, dass zwischen Instanziierungen einer Konstruktion im freien und fixierten Gebrauch ein fließender Übergang, ein Kontinuum besteht. Auf diese Weise kann der konstruktionsgrammatische Ansatz mit Elementen der Phraseologie weiter ergänzt bzw. bereichert werden.

Um den Fragen auf den Grund zu gehen, sind für das Französische und das Italienische Belege aus den Korpora des "SketchEngine“2 und für das Deutsche aus DeReKo (das Deutsche Referenzkorpus des Instituts für Deutsche Sprache in Mannheim (IDS)) und des Digitalen Wörterbuchs der Deutschen Sprache (DWDS) gesammelt worden. Da Beispiele für das von uns analysierte Phänomen in den verschiedenen elektronischen Korpora nicht ausreichend vertreten sind (das betrifft überwiegend die französischen und italienischen Korpora), war es notwendig, auch Belege durch eine Google-Suche und zum Teil aus dem alltäglichen Leben heranzuziehen, die die Autoren in der jeweiligen Sprachgemeinschaft gesammelt haben. ${ }^{3}$ Es handelt sich also um ein offenes Korpus, das einerseits aufgrund seiner Größe eine tiefgründige Analyse und Aussagen über Analogien und Unterschiede innerhalb der untersuchten Sprachen ermöglicht, das andererseits jedoch keine genauen Angaben über die Frequenz der analysierten Konstruktion gestattet.

Unser Artikel ist wie folgt strukturiert. Zur besseren Abgrenzung der untersuchten Konstruktion listet Abschnitt 2.1. zuerst einige homonyme Konstruktionen auf, die jedoch nicht Untersuchungsgegenstand dieser Studie sind. Die konstituierenden Bestandteile der kausalen [ADJ+PP]-Struktur werden im Abschnitt 2.2. etwas näher besprochen, während Abschnitt 2.3. sich intensiver mit einigen definitorischen Merkmalen der analysierten Konstruktionen, nämlich dem Kompositionalitätsgrad und dem metaphorischen und/oder metonymischen Gebrauch der kausalen Konstruktion im fixierten Gebrauch, befasst. Die möglichen Vererbungs-

\footnotetext{
2 Der SketchEngine ist ein „,corpus query system incorporating word sketches, one-page, automatic, corpusderived summary of a word's grammatical and collocational behaviour [...] which gives access to large corpora for 42 languages“" (http://www.SketchEngine.co.uk/?page=Website/SketchEngine) [12.05.2017].

${ }^{3}$ Der Quellennachweis wird nach jedem Beleg angegeben. Manchmal sind die Belege von uns exemplarisch formulierte Sätze, die dann von deutschen Muttersprachlern überprüft worden sind (in diesem Fall fehlt ein Quellennachweis).
} 
beziehungen zwischen den verschiedenen Konstruktionen und den Instanziierungen der kausalen Konstruktion werden in Abschnitt 2.4. besprochen. Abschnitt 3 konzentriert sich auf den möglichen phraseologischen Charakter der [ADJ+PP]kausal-Konstruktion und die Unterscheidung zwischen nicht-lexikalisierten und phraseologischen bzw. idiomatischen Konstruktionen mit besonderer Berücksichtigung des Konzepts der Phrasem-Konstruktionen. Dabei wird auf Fragestellungen und Auffassungen aus dem Bereich der Phraseologie näher eingegangen (cf. Burger 2007; Dobrovol'skij 2011; Donalies 2009; Fleischer 1997; Gries 2008; Wulff 2012). Der abschließende Abschnitt 4 fasst alle Elemente der Diskussion noch einmal zusammen und zeigt den Nutzen der Beschreibung der einzelnen Beispiele als Inventar von Konstruktionen.

\section{Syntaktische und semantische Definition der [ADJ+PP]kausal-Konstruktion}

\subsection{Homonyme Konstruktionen}

Wie schon angekündigt, wird die Untersuchung sich hauptsächlich auf kausale Strukturen [ADJ+PP] fokussieren. Neben der kausalen Konstruktion gibt es eine Reihe von (formal) homonymen Strukturen mit den gleichen Konstituenten, die aber keine kausale Bedeutung vermitteln. Zur Illustrierung können wir folgende Beispiele erwähnen:

(6) it. È molto bella di faccia.

Ist sehr schön von Gesicht

,Sie hat ein sehr schönes Gesicht.‘

(7) fr. Léa est douce de caractère. (Beispiel von Léard und Marengo 2005: 395)

Lea ist sanft von Charakter

,Lea hat einen sanften Charakter.

(8) fr. Patricia est fière de son diplôme.

Patricia ist stolz von ihrem Abschluss

,Patricia ist stolz auf ihren Abschluss.

(9) fr. Il est certain de son coup.

Er ist sicher von seinem Schlag

,Er ist sicher, dass es ihm gelingen wird.

Da unser Untersuchungsgegenstand kausale Strukturen betrifft, verweisen wir hier auf die entsprechende Literatur, die sich mit nicht-kausalen Strukturen befasst, u. a. Frei (1939), Léard/Marengo (2005), und Salles (1998). 


\subsection{Konstituenten der kausalen Konstruktion [ADJ+PP] kausal}

Die oben erwähnten Beispiele (1)-(5) sind Instanziierungen einer Konstruktion mit einer syntaktischen Form und einer entsprechenden Bedeutung. Diese Konstruktion lässt sich wie folgt darstellen:

\begin{tabular}{|l|l|}
\hline Form: & {$[$ ADJ + PP $]$ kausal } \\
\hline Bedeutung: & $\begin{array}{l}\text { ein bestimmter Zustand/eine bestimmte Empfindung ergibt sich als Folge einer } \\
\text { gegebenen Ursache bzw. eines gegebenen Grundes }\end{array}$ \\
\hline
\end{tabular}

Tabelle 1: Form und Bedeutung der kausalen Konstruktion $[\mathrm{ADJ}+\mathrm{PP}]_{\text {kausal }}$

Beide Seiten bilden ein Form-Bedeutungs-Paar, das durch die Prinzipien der NichtModularität und der Nicht-Derivation gekennzeichnet ist. Form-Bedeutungs-Paare sind nichtmodular, da sie ,in einer untrennbaren Beziehung (in Konstruktionen) zueinander stehen und daher nur in ihrer Einheit (in Konstruktionen) analysiert werden können“" (Smirnova und Mortelmans 2010: 134). Das Prinzip der Nicht-Derivation besagt, dass „Konstruktionen [...] nicht voneinander abgeleitet [sind], sondern [...] nebeneinander als relativ selbständige Einheiten [existieren]“" (Smirnova und Mortelmans 2010: 134).

Als Form-Bedeutungs-Paar bringt die untersuchte Konstruktion eine kausale Bedeutung zum Ausdruck (cf. Laptieva 2017). Kausale Bedeutung oder Kausalität wird hier in Anlehnung an Ballestracci (2011: 1) als ,die Verknüpfung von Ursachen, Motiven und Gründen mit ihren entsprechenden Folgen, Auswirkungen und Effekten“ verstanden. Radden/Dirven (2008: 327) spezifizieren Kausalität noch etwas weiter: „Causes are situations which trigger off another physical or psychological situation as their effect. Causes can be paraphrased by because of, as a result of, and due to."

Die Präpositionalphrase drückt die Ursache für den Effekt/für die Wirkung aus, die mit dem Adjektiv vermittelt wird:

(10) fr. Il est triste de la perte de sa mère.

Er ist traurig von dem Verlust von seiner Mutter

,Er ist traurig über den Verlust seiner Mutter.

(11) fr. [...] Elle était rouge de colère. (SketchEngine, 538904)

Sie war rot von Wut

,[...] Sie war rot vor Wut.

(12) it. Pino è diventato rosso dalla rabbia

(http://italingua.ning.com/profiles/blogs/voglio-diventare-famoso)

Pino ist geworden rot von der Wut

,Pino ist rot vor Wut geworden.

Der Verlust der Mutter (10), die Wut in (11) und (12) bilden die Ursache für den Zustand der Traurigkeit (10) oder der roten Farbe (im Gesicht) (Beispiele (11) und (12)). Wie zu beobachten ist, tritt bei der Struktur [ADJ+PP] kausal oft ein Farbadjektiv auf. Dieses Adjektiv entspricht meistens einer der elf von Berlin und Kay (1969) definierten Grundfarben; anhand einer Ana- 
lyse in unserem Korpus lässt sich sagen, dass überwiegend die Farben ${ }^{4}$ Weiß, Rot, Grün, Gelb, Blau, und Schwarz in der Kausalkonstruktion im Deutschen, Französischen und Italienischen vorkommen. Diese Farben entsprechen ,concepts anchored in certain , universals of human experience ' $[\ldots]$ we project them onto something in our shared environment." (Wierzbicka 1990: 99). Die Selektion einer bestimmten Farbe in der Kausalkonstruktion ist aber stark an die einzelnen Sprachen gebunden (siehe weiter dazu Abschnitt 2.3).

Die PP in $[\mathrm{ADJ}+\mathrm{PP}]_{\text {kausal }}$ wird durch eine Präposition eingeleitet, die in dieser Konstruktion eine Ursache, einen Grund ausdrückt. Radden/Dirven (2008: 328f.) unterscheiden verschiedene mögliche Ursachtypen: direkte, wie etwa in He died of heart-failure, und indirekte, etwa He died from an overdose of drugs (328); weiter gibt es Ursachen, die Emotionen auslösen, etwa I am happy about stricter smoking policies (329) und Emotionen, die Reaktionen auslösen, etwa He went red with anger (329). Wie aus den englischen Beispielen schon ersichtlich wird, ist diese Kategorisierung wichtig für die Wahl der Präposition (siehe auch zur Wahl der englischen Kausalpräpositionen Dirven 1995, 1997; Khoo/Chan/Niu 2002). Auch die von uns untersuchten Sprachen verfügen über verschiedene Präpositionen für den Ausdruck der Kausalität. Im Französischen hat man z. B. die Präposition de oder die Wortverbindung à cause de. In der Konstruktion [ADJ+PP] kausal kommt - wie in den obigen Beispielen bereits gesehen - aber nur de vor. Im Italienischen erscheinen in der untersuchten Konstruktion die Präpositionen $d a$ und $d i{ }^{5} \mathrm{Da}$ verschmilzt mit dem bestimmten Artikel und bildet die so genannte „preposizione articolata“, wie in

(13) it. Mio cognato è diventato rosso dalla rabbia. (SketchEngine, 1316172)

Mein Schwager ist geworden rot von der Wut

,Mein Schwager ist rot vor Wut geworden.

Dagegen wird $d i$ ohne weiteren Artikel gebraucht:

(14) it. Tamy era rosso di rabbia. (SketchEngine, 0900004)

Tamy war rot von Wut

,Tamy war rot vor Wut.

Die deutsche Sprache verfügt über mehrere Präpositionen, um die Kausalität auszudrücken, nämlich aus, wegen, vor und von (siehe u. a. Eisenberg (2004: 195) und Girdenienë (2006: 49) für eine ausführliche Unterscheidung des Gebrauchs der Präpositionen), allerdings kommen nur vor und von in den besprochenen Kausalkonstruktionen vor. Die Präposition aus kann nur in Verbindung mit einer absichtlichen Handlung benutzt werden, wie etwa in Anna schrie aus Wut; Anna ist sich ihres Schreiens ganz bewusst. Der Gebrauch der Präposition vor dagegen setzt voraus, dass die Handlung nicht absichtlich geschieht. Dies erklärt, warum in Anna weinte vor Trauer eher die Präposition vor gebraucht wird. Die Präposition von kann

\footnotetext{
${ }^{4}$ Die Farbbezeichnungen werden großgeschrieben, wenn die tatsächliche Farbe gemeint ist. Wenn das Adjektiv gemeint ist, wird es kleingeschrieben.

${ }^{5}$ Im Italienischen kann auch die Präposition per z. T. erscheinen: il ragazzo diventò rosso per la rabbia [...] Der Junge wurde rot für die Wut ,Der Junge wurde rot vor Wut.' http://www.diagonalley.it/modules.php ?name $=$ Forums $\&$ file $=$ viewtopic $\& p=1817936$ [12.12.2016].
} 
trotz ihrer primären Bedeutung eines Ursprungs auch eine kausale PP einführen. ${ }^{6}$ Allerdings verschmilzt sie dann - genau wie $d a$ im Italienischen - mit dem bestimmten Artikel, wie aus folgendem Beispiel zu ersehen ist:

(15) dt. Seine Nase war schwarz vom Tabak.

$$
[\mathrm{von}+\mathrm{dem}]
$$

Die Nominalgruppe nach der Präposition kann unterschiedlicher Natur sein, sie kann einer physikalischen Ursache entsprechen, wie etwa in

(16) dt. Die Sportler sind rot, die Bläser blau vor Kälte.

(DWDS, Die Zeit, 04.02.1983, Nr. 6)

Aber oft drückt die Nominalgruppe eine Emotion aus,

(17) dt. Frau Häsli wurde aufmerksam und war rot vor Wut.

(DWDS, Ball, Hugo, Flammetti, Berlin: Reiss 1918, S. 9404)

(18) fr. La jeune fille était devenue rouge de honte.

Die junge Frau war geworden rot von Scham

,Die junge Frau war rot vor Scham geworden.

Nachdem wir die verschiedenen Bestandteile der Kausalkonstruktion kurz beschrieben haben, wollen wir uns mit deren Gebrauch im wörtlichen vs. nicht-wörtlichen Sinne befassen.

\subsection{Kompositionalitätsgrad, metonymischer und metaphorischer Gebrauch der Konstruktion $[\mathbf{A D J}+\mathbf{P P}]_{\text {kausal }}$}

Wie bereits in der Einleitung besprochen, entsprechen die analysierten Beispiele einer fest verankerten, schematischen Konstruktion und zwar $[\mathrm{ADJ}+\mathrm{PP}]_{\text {kausal, die semantisch sowohl }}$ kompositionell als auch nicht-kompositionell instanziiert werden kann. In Goldbergs erstem Buch (1995) wird die Eigenschaft der Nicht-Prädiktabilität bzw. der Nicht-Kompositionalität als ausschlaggebend angesehen, um Konstruktionen zu postulieren. Nach diesem Prinzip ergibt sich die Bedeutung einer spezifischen Struktur aus der gesamten Struktur und kann nicht bloß als die Summe ihrer konstituierenden Elemente aufgefasst werden:

According to Construction Grammar, a distinct construction is defined to exist if one or more of its properties are not strictly predictable from knowledge of other constructions existing in grammar: $\mathrm{C}$ is a construction iffdef $\mathrm{C}$ is a form-meaning pair $<\mathrm{Fi}, \mathrm{Si}>$ such that some aspect of Fi or some aspect of $\mathrm{Si}$ is not strictly predictable from C's component parts or from other previously established constructions.

(Goldberg 1995: 4)

In ihrem späteren Buch (2006) ergänzt Goldberg dieses enge Prinzip der Nicht-Prädiktabilität mit dem Prinzip der Verfestigung (in Goldbergs Terminologie ,entrenchment"). Danach gelten auch kompositionelle Strukturen als Konstruktionen im technischen Sinne, wenn sie in einer bestimmten Sprache verfestigt sind, was anhand des Konzepts der Frequenz festgestellt

\footnotetext{
${ }^{6}$ Die von uns beschriebenen Präpositionen sind in allen drei Sprachen polysem. So kann frz. de nicht nur eine Ursache, sondern auch eine Quelle, einen Ursprung, oder eine Zugehörigkeit ausdrücken (siehe Grevisse 1936: 540).
} 
werden kann: „In addition, patterns are stored as constructions even if they are fully predictable as long as they occur with sufficient frequency (Goldberg 2006: 5)“".

Rostila (2011: 265) präzisiert dieses Prinzip, indem er von „wiederkehrenden Formen“ redet: „Wenn man einen bestimmten sprachlichen Bereich konstruktionsgrammatisch erfassen will, gilt es, wiederkehrende Formen zu ermitteln, deren Bedeutung gleich bleibt. Dies folgt selbstverständlich aus dem Status von Konstruktionen als Form-Bedeutungs-Paaren“.

Je nach Kompositionalitätsgrad lassen sich die von uns analysierten Instanziierungen der schematischen Konstruktion $[\mathrm{ADJ}+\mathrm{PP}]_{\text {kausal }}$ in verschiedene Subkategorien klassifizieren. Bei folgenden Beispielen

(19) dt. Ihre Hände waren blau vor Kälte.

(20) fr. La jeune fille était devenue rouge de honte.

Die junge Frau war geworden rot von Scham

,Die junge Frau war rot vor Scham geworden.

(21) dt. [...] ihre Gesichter sind rot von der beißenden Kälte.

(DWDS, Die Zeit, 03.06.2009, Nr. 7)

ergibt sich die kausale Bedeutung kompositionell aus den einzelnen Konstituenten der Konstruktion, d. h. dass die PP eine Erklärung für die Farbe bietet: Es ist wörtlich gemeint, dass aufgrund eines extremen Zustandes, d. h. durch die Kälte, die Hände blau (Beispiel (19)) und durch das Schamgefühl (Beispiel (20)) oder durch die beißende Kälte (Beispiel (21)) die Gesichter wirklich rot geworden sind. In den drei Belegen betrifft die Farbveränderung entweder einen Körperteil, nämlich das ganze Gesicht (Beispiel (20) bzw. (21)) oder den ganzen Körper (Beispiel (19)). Obwohl diese Beispielsätze alle gleich kompositionell sind, differenzieren sie sich doch in der Ursachenart voneinander: äußere Ursachen wie extreme Temperaturen in (19) oder (21) oder Ursache emotionaler Natur in (20). Bei diesem letzteren Beispiel ist die Farbe des Gesichts metonymisch als Ort für den Ausdruck von Emotionen wie Scham, Wut, Neid, Eifersucht usw. zu verstehen. Soriano/Valenzuela (2009: 423) erklären die Farbveränderung im Gesicht mit dem Aufsteigen einer Körperflüssigkeit in einem Beispiel wie rot vor Wut etwa folgendermaßen: ,the colour refers to a body fluid that intervenes (or is believed to intervene) in the experience of the emotion at stake. In the case of anger the fluid is blood, which rushes to the neck and face areas when we feel outraged." (Soriano/Valenzuela 2009: 423).

Das obige Beispiel (20) ist durch folgende Metonymie gekennzeichnet: Farbe des Gesichts für physischen Zustand. Nach Niemeier (1998: 126) gehören Farben zu einem sehr produktiven metonymischen Bereich, was im Folgenden weiter deutlich wird:

(22) fr. [...] ils étaient tous pâles de terreur et certains ont commencé à vomir (SketchEngine, 05302)

Sie waren alle bleich von Angst und einige haben angefangen zu übergeben

,[...] Sie waren alle bleich vor Angst und einige haben angefangen, sich zu übergeben.

(23) fr. Il était jaune d'indigestion. 
Er war gelb von Magenverstimmung

,Er war gelb von einer Magenverstimmung. ‘

In einigen wenigen Fällen kann jedoch ein und dasselbe Beispiel einer kausalen Struktur mit einem Farbadjektiv kompositionell oder nicht-kompositionell verstanden werden. Betrachten wir noch einmal das oben erwähnte italienische Beispiel:

(3) it. Sono rosso di rabbia.

Bin rot von Wut

,Ich bin rot vor Wut.

In seiner wörtlichen Lesart würde in Beispiel (3) - genauso gilt dies für das Französische (siehe etwa Beispiel (11)) und das Deutsche (Beispiel (17)) - die tatsächliche rote Gesichtsfarbe (verursacht durch Wut) gemeint sein. Es wird dann einfach eine kausale Bedeutung zum Ausdruck gebracht. Aber das gleiche Beispiel kann auch nicht-kompositionell verstanden werden, wenn es auf einen erregten Zustand verweist. In dieser Lesart hat rot vor Wut nichts mit einer wortwörtlichen Farbveränderung zu tun, es wird vielmehr als Intensivierung gebraucht: Es wird mittels einer Metapher auf einen extremen emotionalen Zustand hingewiesen. Häufig wird die wörtliche Lesart erst dann disambiguiert, wenn im Satz ausdrücklich gesagt wird, dass sich vor lauter Aufregung das Gesicht färbt:

(24) it. Aveva il viso rosso di rabbia e ansimava. (SketchEngine, 1454782)

Hatte das Gesicht rot von Wut und ächzte

,Er hatte das Gesicht rot vor Wut und ächzte.

Diese auf einer Metonymie basierende Metapher ist in der wissenschaftlichen Literatur unter dem Konzept der ,Metaphtonymie“ bekannt (siehe dazu unter anderem Goossens 1990). Sie drückt eine Intensivierung aus und steht in dem Beispiel für das Konzept ,sehr wütend'. In seiner Beschreibung von Metaphern und Emotionen weist Kövecses (2000) schon auf die beliebte Metapher ,anger is a hot fluid in a container“ oder „redness in the face stands for anger" (2000: 142) und betont, dass konzeptuelle Metapher und Metonymie eine wichtige Rolle in der Konzeptualisierung von Wut im Englischen und in anderen Sprachen wie im Chinesischen, Ungarischen, oder Japanischen spielen. Nach Kövecses ermöglicht es die Konzeptualisierung der Wut in Termini der Container-Metapher, sehr viele unterschiedliche Aspekte und Eigenschaften der Wut-Emotion zu erfassen: als Intensität (ausgedrückt im Englischen mit filled with), als Kontrolle (Engl. contain), als Kontrollverlust (Engl. could not keep inside), als Gefahr (Engl. brim with), usw. (Kövecses 2000: 147). Auch in den obigen Beispielen mit einem Farbadjektiv wird der menschliche Körper als ein Container betrachtet, in dem eine bestimmte Flüssigkeit aufsteigt.

In seiner nicht-kompositionellen Lesart ist die Struktur rot vor Wut als eine einheitliche Bedeutungseinheit zu interpretieren; die drei konstituierenden Sprachzeichen sind in dieser Lesart fixiert und somit lexikalisiert, auch wenn die Konstruktion ursprünglich durch Metonymie motiviert ist.

Ebenfalls motiviert, jedoch weniger transparent für die Sprecher ist der Gebrauch des Farbadjektivs gelb bzw. grün in Verbindung mit Gefühlen wie Neid und Eifersucht im Deutschen und Italienischen: 
(25) dt. Doch eines Tages antwortete der Spiegel zum Entsetzen der Königin: „Frau Königin, Ihr seid die Schönste hier, aber Schneewittchen ist tausendmal schöner als Ihr! “. Da wurde die Königin grün und gelb vor Neid.

(DeReKo, St. Galler Tagblatt, 09.01.1999, Ressort: WV-UZW

(Abk.); „Schneewittchen“ im Uzwiler Gemeindesaal)

(26) it. Maria è gialla/verde di gelosia.

Maria ist gelb/grün von Eifersucht

,Maria ist gelb/grün vor Eifersucht.

In Verbindung mit Körperflüssigkeiten spielt die gelbe Farbe sicherlich eine Rolle, und zwar zur Kennzeichnung der Galle, der in der Leber produzierten Flüssigkeit. Dies kann der Ursprung der Verbindung von Gelb mit Neid sein; da aber der Übergang von Gelb zu Grün ziemlich leicht ist, ist die alternative Okkurrenz beider Farben in der Wendung motiviert. $\mathrm{Zu}$ beachten ist jedoch, dass die Wahl der Farbadjektive sprachenspezifisch stattfindet. Nach einer Überprüfung der Belege in den Korpora und nach einer Sprecher-Befragung scheint es, dass die Auswahl zwischen Gelb und Grün im Deutschen und im Italienischen ziemlich arbiträr erfolgt, allerdings beobachtet man eine Präferenz für die Farbe Grün im Italienischen und für die Farbe Gelb im Deutschen im Zusammenhang mit Neid und Eifersucht. Die französische Sprache dagegen verbindet fast ausschließlich die Farbe Grün mit denselben Emotionen, auch wenn Beispiele mit dem Farbadjektiv Gelb sporadisch auch zu finden sind: ${ }^{7}$

(27) fr. Marie est verte de jalousie.

Maria ist grün von Eifersucht

,Maria ist gelb vor Eifersucht.

De Knop (2013) untersucht die Präferenzen in der Farbwahl in den deutschen und französischen Kausalkonstruktionen. Sie kommt zu dem Schluss, dass Grün eine dominante Farbe in den französischen kausalen Konstruktionen ist, während Deutsch Gelb und auch Weiß in derselben Konstruktion privilegiert. ${ }^{8}$ In den französischen Kausalkonstruktionen konkurriert die Farbe Grün sogar mit der Farbe Rot für Wut-Emotionen, etwa

(28) fr. [...] des fois, j'en deviens vert de colère. (SketchEngine, 12930)

Manchmal, ich davon werde grün von Wut

,[...] manchmal werde ich davon grün vor Wut.

Auch im Deutschen und Italienischen ist dieselbe Farbe Grün in Verbindung mit Wut zu finden:

(29) dt. Sonst wird er nicht nur grün vor Wut, sondern auch vor Neid auf die erfolgreicheren Super-Kollegen aus dem Hause Marvel.

(http://www.stern.de/kultur/film/-der-unglaubliche-hulk--gruene-gefahr-reloaded3858966.html)

(30) it. Quando si arabbiava diventava verde di rabbia. (SketchEngine, 1425425)

\footnotetext{
${ }^{7}$ Siehe etwa: http://www.legrenierdebibiane.com/participez/Expressions/couleurs.html. [12.03.2016]

${ }^{8}$ Auch Blau ist im Französischen eine beliebte Farbe (für weitere Details, Beispiele und die statistischen Belege, siehe De Knop 2013).
} 
Wenn sich ärgerte wurde grün von Wut

,Wenn er sich ärgerte, wurde er grün vor Wut.

Eine Erklärung für die Dominanz der Farbe Grün in solchen Konstruktionen könnte darin bestehen, dass die Sprecher viele negative Emotionen mit der Gallenflüssigkeit in Verbindung bringen, die ursprünglich als grünlich wahrgenommen wurde. ${ }^{9}$

In einigen sporadischen Fällen ist die Verbindung des Farbadjektivs mit dem darauffolgenden Emotionsausdruck überhaupt nicht mehr transparent bzw. durch Metonymie erklärbar, obwohl die Kausalkomponente deutlich ist, wie etwa mit Beispiel (31) illustriert wird: ${ }^{10}$

(31) it. Sono nero dalla rabbia oggi.

Bin schwarz von der Wut heute

,Ich bin sehr sauer/wütend heute.

Nach Dobrovol'skij/Piirainen (2002: 250f.) ist Schwarz die Farbe mit der negativsten Konnotation, denn ,es ist die Farbe der Dunkelheit und der Nacht [...]. Tod, Trauer und Hoffnungslosigkeit werden mit Schwarz assoziiert [...]“. Die Autoren führen weiter aus, dass diese Farbe auch einen negativen psychischen Zustand symbolisiert. Außerdem erwähnen Soriano/Valenzuela (2009: 424) in ihrem Artikel über Assoziationen mit spanischen Farbtermini die russischen Beispiele „black envy“ (,schwarzer Neid“) und „white envy“ (,weißer Neid‘), bei denen die negativen (im Falle von „,black“) oder positiven (bei „white“) Assoziationen durch Metaphern motiviert sind. Im Italienischen gibt es ein ähnliches Beispiel:

(32) it. Sono nero oggi.

Bin schwarz heute

,Ich bin sehr sauer/wütend/schlecht drauf heute.

Der Gebrauch des Adjektivs schwarz in der Kausalkonstruktion scheint doch sehr idiosynkratisch zu sein und gebunden an eine bestimmte Sprache, in diesem Falle an Italienisch. ${ }^{11}$

\subsection{Vererbungsbeziehungen}

Die Besprechung der Beispiele nach dem Kompositionalitätsgrad, nach dem metonymischen oder metaphorischen Gebrauch im vorherigen Abschnitt hat die große Variationsbreite veranschaulicht und deutlich gemacht, dass man statt der Dichotomie ,wörtliche vs. phraseologische Struktur“ bzw. „freie Syntax vs. Lexikon“ eher von einem Kontinuum zwischen den Instanziierungen derselben Konstruktion reden sollte. Genauso wie Lexeme können Konstruktionen polysem sein, d. h. ein und dieselbe Konstruktion kann verschiedene miteinander ver-

\footnotetext{
${ }^{9}$ Die Farbe Gelb wird dagegen mit dem Schwindelgefühl assoziiert, das eventuell auch durch Neid ausgelöst werden kann (Gelb wird außerdem häufig auch in Verbindung mit Krankheit gebraucht). http://www.wissen.de/woher-kommen-die-redewendungen-gelb-vor-neid-und-sich-schwarz-aergern [12.12.2016].

10 Auch im Deutschen kommt schwarz in Verbindung mit Ärger bzw. Wut vor (siehe z. B. auch sich schwarz ärgern), wobei diese Konstruktion (nach einer Überprüfung in DeReKo) nicht so frequent wie im Italienischen zu sein scheint: Beim Warten auf den Telekominstallateur wurde schon mancher schwarz vor Ärger. (DeReKo, Hamburger Morgen Post, 13.05.2007).

${ }^{11}$ Staffeld (cf. 2011: 139) zeigt, dass Schwarz im Deutschen neben den oben beschriebenen Assoziationen auch zur Bedeutung für ,illegal beiträgt‘ (z. B. schwarzfahren, Schwarzmarkt usw.).
} 
bundene Bedeutungslesarten zum Ausdruck bringen. Dies trifft auch auf die hier untersuchte Struktur $[\mathrm{ADJ}+\mathrm{PP}]_{\mathrm{kausal}} \mathrm{Zu}$, wie wir schon mit Beispiel (3) it. Sono rosso di rabbia (,Ich bin rot vor Wut') feststellen konnten: es kann sowohl wörtlich als auch metonymisch verstanden werden. Die verschiedenen Konstruktionen einer Sprache bilden kein ungeordnetes Inventar, sondern sind nach Goldberg $(1995,2006)$ durch so genannte Vererbungsbeziehungen (Englisch inheritance links) zu einem strukturierten Netzwerk miteinander verbunden. Vererbungsbeziehungen motivieren die Relationen zwischen Konstruktionen, sie beruhen auf einer Dominanzbeziehung zwischen den Konstruktionen, d. h. dass eine bestimmte Konstruktion (K1) ihre Eigenschaften an eine weitere Konstruktion (K2) vererbt, was zur Folge hat, dass K2 von K1 dominiert wird. Goldberg (1995: 79) unterscheidet vier Typen von Vererbungsbeziehungen: die Polysemie-Beziehung, die Teil-von-Beziehung, die Beispiel-von-Beziehung und die metaphorische Beziehung (für die Übersetzung der englischen Termini ins Deutsche orientieren wir uns an Ziem/Lasch 2013: 98-102). Ein Beleg wie (33):

(33) fr. Il est triste de la perte de sa mère.

Er ist traurig von dem Verlust von seiner Mutter

,Er ist traurig über den Verlust seiner Mutter.‘

kann als prototypisches Beispiel für die $[\mathrm{ADJ}+\mathrm{PP}]_{\text {kausal-Konstruktion betrachtet werden, da er }}$ in seiner freien Verwendung aktualisiert wird und als solcher verfestigt ist. Beispiel (3) it. Sono rosso di rabbia. (,Ich bin rot vor Wut.') ist durch eine Beispiel-von-Beziehung mit dem prototypischen Beispiel von Kausalkonstruktion (33) verbunden. Nach Goldberg liegt eine Beispiel-von-Beziehung dann vor, wenn z. B. eine Konstruktion eine lexikalisch spezifizierte Variante einer anderen Konstruktion darstellt. Sie sei also ein Spezialfall dieser Konstruktion, von der sie die Syntax und Semantik erbt (Goldberg 1995: 79). Das heißt, man hat einerseits die prototypische, schematische, abstrakte und nicht-idiomatische Konstruktion $[\mathrm{ADJ}+\mathrm{PP}]_{\text {kausal, }}$ die auf der lexikalischen Ebene nicht spezifiziert ist. Andererseits gibt es Beispiele mit einem Farbadjektiv wie in (3)-(5), die konkrete phraseologische Instanziierungen (siehe Abschnitt 3) der allgemeinen Kausalkonstruktion sind.

Weitere Vererbungsbeziehungen kennzeichnen unsere Beispiele. Wenn etwa Beispiel (3) nicht-kompositionell, sondern idiomatisch zu verstehen ist, dann ist diese Lesart eine metaphorische Erweiterung der Grundstruktur. Wie Ziem (2014: 57) ausführt, „tragen Goldberg zufolge konzeptuelle Metaphern dazu bei, bestimmte Konstruktionen und Konstruktionsbedeutungen zu lizenzieren [...], die Produktivität einer Konstruktion mit zu bestimmen und sie somit im Sprachwissen bzw. im Konstruktikon der SprachbenutzerInnen zu etablieren“.

Eine Teil-Ganzes-Beziehung verbindet beide oben schon erwähnte Beispiele:

(34) it. Sono nero dalla rabbia oggi.

Bin schwarz von der Wut heute

,Ich bin sehr sauer/wütend/schlecht drauf heute.

(35) it. Sono nero oggi.

Bin schwarz heute

,Ich bin sehr sauer/wütend/schlecht drauf. 
Nach Goldberg (1995: 78) liegt diese Vererbungsbeziehung dann vor, wenn eine Konstruktion Teil einer anderen Konstruktion ist. Gleichzeitig aber existieren beide Konstruktionen unabhängig voneinander (cf. auch Ziem/Lasch 2013: 100). Die Konstruktion in (35) ist Teil der

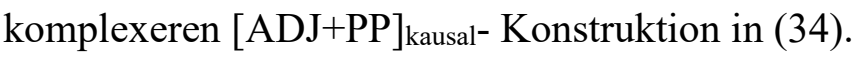

\section{Der phraseologische Charakter der [ADJ+PP] kausal -Konstruktion}

\subsection{Terminologische Fragen}

Da wir immer wieder mit phraseologischen Einheiten konfrontiert werden, scheint es uns wichtig, an dieser Stelle kurz auf einige terminologische Fragestellungen im Bereich der Phraseologie einzugehen. Konstruktionen wie dt. rot vor Wut sein und ihre Entsprechungen, it. essere rosso di rabbia, fr. être rouge de colère oder it. essere nero ,schlechte Laune haben“ (wörtlich: ,schwarz sein') sind sogenannte Phraseologismen und als solche traditionellerweise Untersuchungsgegenstand der Phraseologie. Phraseologismen sind in der Fachliteratur unterschiedlich beschrieben worden (vergleiche unter anderem etwa Burger 2010; Donalies 2009; Fleischer 1997; Gries 2008; oder Wulff 2012). "They can range from free combinations to restricted collocations, figurative idioms, and pure idioms" (Wulff 2012: 292). Sie bilden ein "subset of phrases maximally conventionalized" (Wulff 2012: 292). Gries (2008) diskutiert eine Reihe definierender Eigenschaften der Phraseologismen. Das Hauptcharakteristikum in der Definition eines Phraseologismus ist eine ,semantic unity, but not non-compositional semantics" (Gries 2008: 3). Dies betrifft auch unsere Beispiele - wie wir schon feststellen konnten. Zur Kennzeichnung wörtlicher und nichtwörtlicher fixierter Ausdrücke konkurriert der Terminus „Phraseologismus“ in der Fachliteratur mit einer Reihe anderer alternativer Termini, etwa „phraseologische Einheit“, „Idiom“, „Phrasem“, ,idiomatischer Ausdruck“, usw. (siehe weiter dazu Donalies 2009: 30). Bezugnehmend auf Fleischer (1997: 3), werden wir den Terminus ,Phraseologismus' als generischen Terminus für fixierte wörtliche oder nichtwörtliche Wortkombinationen benutzen und ,Idiom‘ spezifisch für nicht-wörtliche, voll lexikalisierte Wortkombinationen (siehe auch Burger 2010: 37).

In diesem Kontext wollen wir etwas näher auf eine besondere idiomatische Wendung eingehen, und zwar auf den belgisch-französischen Ausdruck être bleu de (siehe dazu http://www.bdlp.org/resultats.asp?base=BE, [02.08.16]), wie etwa in

(36) fr. Pierre est bleu de musique punk.

Peter ist blau von Musik Punk

,Peter liebt Punkmusik/ist verrückt nach Punkmusik. ‘

Dieser Beleg enthält dieselben Konstituenten wie die oben erwähnten Beispiele von Kausalkonstruktionen mit Farbadjektiven, das heißt, er hat die Form [ADJ+PP]. Allerdings trägt er keine kausale Bedeutung, wie auf den ersten Blick zu erwarten wäre. Bleu bezieht sich hier nicht auf die Farbe des Gesichts, sondern steht zusammen mit der Präposition de als Metapher (cf. Derrig 1978; Sweetser 1990) für eine Leidenschaft, eine Passion. Auf Deutsch könnte man es mit ,verrückt nach etwas sein' wiedergeben. Die zugrundeliegende konzeptuelle Metapher lautet ,etwas lieben ist blau sein ${ }^{6}{ }^{12}$ Da die zugrundeliegende Motivation nicht mehr zu

\footnotetext{
12 Nach der Theorie der konzeptuellen Metapher von Lakoff/Johnson (1980) werden konzeptuelle Metaphern in verschiedenen Sprachen unterschiedlich realisiert. Wir möchten an dieser Stelle auf den deutschen Ausdruck
}

ISSN 1615-3014 
erkennen ist, ist Beispiel (36) idiomatisch und unterscheidet sich von den folgenden, kausalen Beispielen mit demselben Farbadjektiv bleu/blau:

(37) dt. Die Sportler sind rot, die Bläser blau vor Kälte.

(DWDS, Die Zeit, 04.02.1983, Nr. 6)

(38) fr. Tout le monde était malade, blessé, les enfants étaient bleus de froid.

(SketchEngine, 12255)

Alle waren krank, verletzt, die Kinder waren blau von Kälte

,Alle waren krank, verletzt, die Kinder waren blau vor Kälte.

In diesen Beispielen weist das Gesicht wegen der Kälte einen bläulichen Schimmer auf. Während die Bedeutung der Belege in (37) und (38) kompositioneller Natur ist, ist die Bedeutung von (36) idiomatisch und voll lexikalisiert. Aus diesem Grunde gehören Beispiel (36) sowie die anderen Wendungen im fixierten Gebrauch mit der Struktur $\left[\mathrm{ADJ}_{\text {farbe }}+\mathrm{PP}\right]_{\text {kausal }}$ zum Bereich der Phraseologie und sind nicht Forschungsgegenstand der Syntax, die sich traditionellerweise mit der freien Kombination der Wörter in einem Sprachsystem befasst.

\subsection{Phrasem-Konstruktionen}

Die bisherige Beschreibung hat sich hauptsächlich auf die phraseologischen Aktualisierungen der Kausalkonstruktion bezogen, die ein Farbadjektiv enthalten. Allerdings sind auch Instanziierungen möglich, die in ihrer Struktur kein Farbadjektiv aufweisen, wie etwa in dem schon erwähnten Beispiel (33) oder in den folgenden Belegen (39)-(42):

(39) fr. Cette ville est malade, m'écrit Durito, malade de solitude et de peur. (SketchEngine, 02953)

Diese Stadt ist krank, mir schreibt Durito, krank von Einsamkeit und von Angst ,Diese Stadt ist krank, schreibt mir Durito, krank vor Einsamkeit und vor Angst.

(40) dt. Ich werde verrückt vor Eifersucht. (http://forum.gofeminin.de /forum/ couple1/f5651_couple1-ich-werde-verruckt-vor-eifersucht.html)

(41) fr. Il est mort d'ennui.

Er ist tot von Langeweile

,Er ist tot vor Langeweile/Er langweilt sich zu Tode.

(42) it. Sono pazza di gelosia.

Bin verrückt von Eifersucht

,Ich bin verrückt vor Eifersucht.

Diese Beispiele können nicht wörtlich verstanden werden: Es handelt sich nämlich um teilidiomatisierte Instanziierungen, mit denen primär keine reine Kausalität, sondern eher extreme Zustände ausgedrückt werden. Ihre Kausalkomponente ist jedoch, wenn auch nicht mehr vordergründig, immer noch präsent, ${ }^{13}$ da solche Konstruktionen wie folgt paraphrasiert wer-

\footnotetext{
blau sein aufmerksam machen, der nichts mit dieser konzeptuellen Metapher zu tun hat und der eine ganz andere Bedeutung hat, nämlich ,betrunken sein'.

${ }^{13}$ Aufgrund der verblassten Kausalsemantik verwenden wir bei der Notation unten das Zeichen \pm .
} 
den können: ,X empfindet den Zustand, die Emotion, das Gefühl Y so stark, dass er fast den Eindruck hat, er könnte krank/verrückt werden bzw. sterben. ' Dabei ist mit X ein Lebewesen (in der Regel ein Mensch) und mit Y ein Zustand (Eifersucht, Hunger, Neugier usw.) gemeint. Der extreme Charakter ergibt sich bei dieser Konstruktion aus dem Zusammenkommen von Y in Verbindung mit Adjektiven, die etwas Exzessives zum Ausdruck bringen. Solche Strukturen sind insofern motiviert, als ihre Bedeutung heute den Sprechern als transparent erscheint, da sie die semantische Beziehung zwischen wörtlicher und phraseologischer Bedeutung noch erkennen können. Aber aufgrund eines Lexikalisierungsprozesses sind diese Wendungen nicht kompositionell zu verstehen, sie werden meistens im übertragenen Sinne verstanden. In Vergangenheitsformen ist dies in Verbindung mit dem Konzept Tod vor allem an dem Auftreten von Adverbien wie dt. fast bzw. halb, fr. presque und it. quasi zu erkennen (siehe das französische und italienische Beispiel und die jeweilige deutsche Übersetzung in (43) und (44) hier unten). Man kann solche Konstruktionen formal wie folgt darstellen:

\begin{tabular}{|l|l|}
\hline Form: & {$[\mathrm{X}$ KOPULA ADJ } \\
\hline Bextr. Zustand + Präp $\left.p_{\text {dt. vor/fr.de/it.di }}+Y_{\text {Zustand/Gefüh/Bedürfnis }}\right]_{\text {kausal } \pm}$ \\
\hline $\begin{array}{l}\text { X empfindet einen extremen Zustand als Folge von Y } \\
\text { (Ursache=Zustand/Emotion/Gefühl) }\end{array}$ \\
\hline
\end{tabular}

Tabelle 2: Kausale Phrasem-Konstruktion

Bei dieser Konstruktion sind einige Positionen relativ frei zu besetzen. Einerseits ist das in dieser Konstruktion auftretende Adjektiv bedingt austauschbar, sofern es einen extremen $\mathrm{Zu}$ stand ausdrückt, wie etwa das Totsein (dt. tot/fr. mort/it. morto), das Verrücktsein (dt. verrückt/fr. fou/it. pazzo), oder das Kranksein (dt. krank/fr. malade/it. malato). Andererseits ist eine größere Variation auf der Ebene des in der PP enthaltenen Nomens möglich, wobei es einen Zustand, eine Emotion oder ein Bedürfnis als mögliche Ursache für das extreme Empfinden von $\mathrm{X}$ zum Ausdruck bringen muss:

(43) fr. Il est (presque) mort de faim/de soif/de peur/de fatigue

Er ist fast tot von Hunger/Durst/Angst/Müdigkeit

,Er ist fast/halb tot vor Hunger/Durst/Müdigkeit. ‘

(44) it. (lui) è (quasi) morto di fame/di sete/di paura /di stanchezza

(Er) ist tot vor Hunger/Durst/Angst/Müdigkeit

,Er ist fast/halb tot vor Hunger/Durst/Müdigkeit.

$\mathrm{Zu}$ beobachten ist jedoch, dass diese Konstruktionen im Französischen und Italienischen viel häufiger auftreten als im Deutschen, ${ }^{14}$ das sich eher Paraphrasen wie Er ist fast vor Hunger/Durst/Angst/Müdigkeit gestorben oder Er hat großen Hunger/Durst/Angst bzw. Er ist sehr müde bedient.

In der deutschen Phraseologieforschung werden Strukturen wie in (43) und (44) traditionellerweise „Phraseoschablonen“ (cf. Fleischers 1997: 130) genannt. Darunter versteht man solche Fügungen, die eine gewisse syntaktische Festigkeit aufweisen, die aber bedingt lexikalisch variabel sind, dabei aber ein Modell bilden. Zur Kennzeichnung solcher Strukturen benutzt Dobrovol'skij (2011: 114) den Terminus „Phrasem-Konstruktion“ (PhK), den er wie

\footnotetext{
14 Obwohl es sich lohnen würde, eine Frequenzanalyse zwischen den drei untersuchten Sprachen durchzuführen, konnte dies im Rahmen dieser Studie nicht realisiert werden.
} 
folgt definiert: „PhK können als Konstruktionen definiert werden, die als Ganzes eine lexikalische Bedeutung haben, wobei bestimmte Positionen in ihrer syntaktischen Struktur lexikalisch besetzt sind, während andere Slots darstellen, die gefüllt werden müssen." (Dobrovol'skij 2011: 114)

Sowohl Dobrovol'skij (2011) als auch Fleischer (1997) rechnen dazu Fügungen wie: ${ }^{15}$

$\begin{array}{ll}{[\mathrm{X} \text { KOPULA X] }} & \text { sicher ist sicher / Geschenk ist Geschenk } \\ {[\mathrm{N} 1 \mathrm{sg} \text { der } \mathrm{N} 1 \mathrm{pl}]} & \text { Spiel der Spiele } \\ {[\mathrm{N} 1 \text { hin, N1 her }]} & \text { Freundschaft hin, Freundschaft her } \\ {[\text { es/das } \mathrm{IST} \text { zum Ninf] }} & \text { Es ist zum Verrücktwerden } \\ {[\mathrm{Xnom} \text { und } \mathrm{Y}]} & \text { Ich und die Männer? }\end{array}$

Diese Strukturen sind partiell schematisch, da sie nur eine begrenzte Anzahl von lexikalischen Varianten zulassen. Zur Kennzeichnung solcher Konstruktionen verwendet man in der Konstruktionsgrammatik Termini wie „formal idioms“ Fillmore/Kay/O'Connor (1988: 505f.) oder „schematic idioms“ Croft/Cruse (2004: 234). Im Folgenden übernehmen wir Dobrovol'skijs Terminologie, da sie u. E. am besten die Idee des existierenden Kontinuums zwischen fixierten Phraseologismen (Forschungsgegenstand der Phraseologie) und den Argument-StrukturKonstruktionen im Sinne Goldbergs (1995) (Forschungsgegenstand der Syntax) kennzeichnet. Dieses Kontinuum ist auch bei der Beziehung zwischen den untersuchten Konstruktionen ziemlich deutlich (siehe weiter dazu Abschnitt 4). Nach dem erwähnten Prinzip der Nicht-

Derivation wird die Phk $\left[X \quad\right.$ KOPULA $\mathrm{ADJ}_{\text {extr. }}$ Zustand+Präp $\mathrm{dt}_{\text {d. }}$ vor $/$ fr.de/

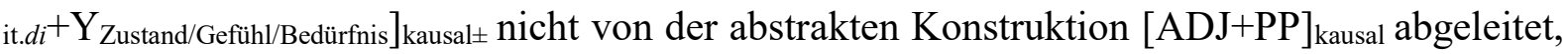
sondern die zwei Konstruktionen koexistieren nebeneinander. Aber sie sind durch metaphorische Links (cf. hierzu Goldberg (1995: 81)) miteinander verbunden, da die Idee einer Kausalität - wenn auch nicht mehr vordergründig - noch metaphorisch rekonstruierbar ist.

\subsection{Kontrastive Analyse der Phrasem-Konstruktionen fr. [X KOPULA mort de Y] bzw. it. [X KOPULA morto di Y] vs. [X KOPULA tot vor Y]}

Wie bereits oben erwähnt, kommt die besprochene Phk im Deutschen - im Gegensatz zum Französischen und Italienischen - nicht so häufig vor. Dies lässt sich dadurch erklären, dass die deutsche Sprache viel stärker differenziert zwischen dem Vorgang des Sterbens und dem Resultat, nämlich dem Tod, was durch unterschiedliche Ausdrucksweisen ([X ist gestorben an

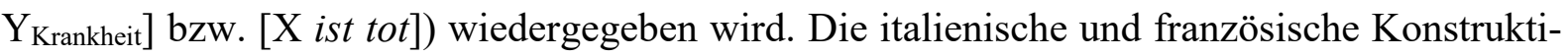
on [morto di] bzw. [mort de] dagegen ist polysem, da sie in dieser Form sowohl das Resultat (siehe Beispiel (46) hierunter) als auch den Sterbevorgang (Beispiel 47) ausdrücken kann.

(46) it. (Lui) è morto

(Er) ist tot

,Er ist tot.

(47) it. (Lui) è morto di cancro

(Er) ist gestorben an Krebs

15 Wir verwenden hier Dobrovol'skijs (2011) Notation. 
,Er ist an Krebs gestorben.

Die Mehrdeutigkeit im Französischen und Italienischen ist dadurch bedingt, dass ein und dieselbe Form zu zwei unterschiedlichen Wortarten gerechnet werden kann:

(a) entweder einem Partizip Perfekt von etwa fr. mourir/it. morire (fr. mort/it. morto) für den Ausdruck des Vorgangs, wie etwa in Beispiel (47) oder in (48):

(48) fr. En Afrique, de nombreux enfants sont morts de faim et de soif.

In Afrika viele Kinder sind gestorben von Hunger und von Durst

,In Afrika sind viele Kinder an Hunger und Durst gestorben.

(b) oder fr. mort/it. morto entsprechen einem Adjektiv (das ursprünglich aus einem Partizip Perfekt entstanden ist) für den Ausdruck des Zustands. Hier betrachten wir fr. mort/it. morto als dieser Wortart zugehörig. Wie oben ausgeführt, kann das Adjektiv in der PhK auch metaphorisch für einen extremen Zustand verwendet werden.

Eine weitere Erklärung für die geringere Häufigkeit des deutschen Ausdrucks [X ist tot vor $\mathrm{Y}$ ] hängt von den beliebten Versprachlichungsmöglichkeiten im Deutschen ab. Im Gegensatz zu den romanischen Sprachen, die eine analytische Ausdrucksweise mit syntaktischen Strukturen mit Nominalphrasen bevorzugen, privilegiert die deutsche Sprache eher synthetische Ausdrucksweisen (siehe Hinrichs 2004, Primus 1997, Siemund 2004, und Zimmer 1996: 37), etwa mit Komposita oder Ableitungen. Dies ist ein beliebtes Versprachlichungsmittel, vor allem für den Ausdruck eines extremen Zustands, wie etwa in saublöd, bärenstark, Bombenlaune, Höllendurst, lammfromm, usw. Diese Bildungen nennt Lohde (2006: 64) Augmentativa oder Vergleichsbildungen. Die Vorliebe für Komposita trifft auch auf die besprochenen Beispiele zu, etwa

(49) fr. Il est mort de faim $\rightarrow$ Er hat einen Riesenhunger/Bärenhunger

(50) fr. Il est mort de soif $\rightarrow$ Er hat einen Riesendurst/Höllendurst

(51) fr. Il est mort de fatigue $\rightarrow$ Er ist todmüde

(52) fr. Il est mort de peur $\rightarrow$ Er hat Todesangst (jedoch auch: Er stirbt vor Angst).

Das Sprachzeichen Tod fungiert häufig als erste Konstituente eines augmentativen Kompositums, wie etwa in todkrank, todunglücklich, todtraurig, todernst, usw. Somit wird im Deutschen durch andere Sprachmittel dasselbe Konzept realisiert wie im Französischen und Italienischen, wo eine analytischere Ausdrucksweise privilegiert wird. An dieser Stelle können wir nicht weiter im Detail auf dieses Thema eingehen, verweisen aber auf Zeschel (2012) und De Knop (2015).

Die adjektivischen PhK dt. [tot vor], fr. [mort de] bzw. it. [morto di] sind mit einer anderen, verbalen Konstruktion bzw. PhK verbunden, und zwar mit dt. [sterben vor], fr. [mourir de] bzw. it. [morire di], die in den drei von uns beschriebenen Sprachen (also auch im Deutschen!) ziemlich produktiv ist. Auch hier kann diese Struktur wörtlich oder im übertragenen Sinne verstanden werden, wie im Folgenden illustriert wird:

(53) a. fr. mourir d'un cancer

b. it. morire di cancro 
c. dt. an Krebs sterben

(54) a. fr. mourir de douleur/de la peine

b. it. morire di dolore/di dispiacere 16

c. dt. vor Schmerz/Kummer sterben.

In (53) wird wörtlich die tatsächliche Ursache des Sterbens in der PP angegeben. Die PP in (54) drückt dagegen ein Gefühl, einen Zustand aus, der so stark ist, dass ein Vergleich mit dem Tod konzeptualisiert wird. Zu beobachten ist, dass die deutsche Sprache auch in diesem Fall weiter differenziert, indem verschiedene Präpositionen für den Ausdruck der Kausalität benutzt werden, nämlich an (siehe (53)) für die wörtliche Bedeutung und vor (etwa in (54)) für die übertragene Bedeutung.

Diese Art der Konzeptualisierung ist auch in folgenden teil-idiomatischen Beispielen vorhanden, wobei die Verbindung mit dem Tod noch weniger präsent ist als in den bereits besprochenen Beispielen:

(55) a. fr. mourir de faim/soif/peur/chaleur/froid

b. it. morire di fame/sete/paura/caldo/freddo

c. dt. vor Hunger/Durst/Angst/Hitze/Kälte sterben

(56) a. fr. mourir de curiositéld'ennui/de honte/d'envie/de jalousie

b. it. morire di curiosità/noia/vergogna/invidia/gelosia

c. dt. vor Neugier/Langeweile/Scham/Neid/Eifersucht sterben

Die in (55) und (56) realisierte Konstruktion unterscheidet sich von den Instanziierungen in (53) und (54) durch verschiedene Grade der Konkretheit vs. Abstraktheit. Während in (53) das Verb seine wörtliche Bedeutung beibehält und die deutsche regierte Präposition an die tatsächliche Ursache des Sterbens angibt, wird in (54), (55) und (56) das Konzept des Sterbens metaphorisch für eine extreme Empfindung verwendet. Im Gegensatz zu den Beispielen unter (53) sind (54), (55) und (56) durch Nicht-Kompositionalität gekennzeichnet, da sich ihre Bedeutung nicht aus der Summe ihrer Bestandteile ableiten lässt. Man könnte eine Art Kontinuum postulieren, das von den konkreten syntaktischen Strukturen bei (53) bis zur metaphorischen Verwendung in (56) reicht. Die Instanziierungen in (55) liegen dazwischen, da man zwar vor Hunger, Durst oder Angst sterben kann, aber diese Strukturen werden meistens in unserer westlichen Welt phraseologisch und nicht wörtlich verwendet. Zu beachten ist jedoch, dass man im Deutschen in (54) und (55) bei wörtlicher Bedeutung die Präposition an verwenden würde, während in übertragener Bedeutung die Präposition vor vorkommt.

Interessant ist auch, dass im übertragenen Sinne Sprachzeichen in dieser PhK kombiniert werden können, deren Bedeutungen zuerst mal als inkompatibel gelten würden. Betrachten wir folgende Beispiele:

\footnotetext{
16 Im Italienischen besteht bei vielen Strukturen die Möglichkeit, eine preposizione articolata $(d a+$ Artikel $)$ statt der einfachen Präposition di ohne Bedeutungsänderung zu verwenden: morire di dispiacere / dal dispiacere, morire di curiosità / dalla curiosità, usw. Hier wird immer die einfache Form verwendet.
} 
(57) a. fr. [X meurt du plaisir/du désir de + INF.]

b. it. [X muore dalla voglia/dal desiderio di $+\mathrm{INF}$.]

c. dt. wörtlich: [,X stirbt von der Lust/dem Wunsch + INF.']

(58) a. it. Moriva dal desiderio di andare in vacanza.

b. fr. Il mourait du désir de partir en vacances.

c. dt. wörtlich: ,Er starb an dem Wunsch, in Urlaub zu fahren.‘

Auch hier wird ein extremer Wunsch ausgedrückt, die kausale Bedeutung ist überhaupt nicht mehr präsent. Aber solche $\mathrm{PhK}$ sind auf sehr wenige Abstrakta (etwa it. voglia, desiderio/fr. désir, plaisir) beschränkt und kommen eher selten vor.

\section{$4 \quad$ Abschließende Bemerkungen und Fazit}

In diesem Beitrag haben wir uns mit der syntaktischen Struktur [ADJ+PP] auseinandergesetzt. Die Untersuchung der Beispiele im Rahmen des Konstruktionsmodells hat uns ermöglicht, die Beispielvariation besser zu erfassen. Bevor wir dieses Kapitel schließen, möchten wir auf die in der Einleitung gestellten Fragen zurückkommen und sie kurz beantworten/kommentieren.

(i) Sind die besprochenen Beispiele Instanziierungen derselben Konstruktion oder entsprechen sie vielleicht verschiedenen Konstruktionen?

Die Struktur [ADJ+PP] entspricht unterschiedlichen Konstruktionen (im technischen Sinne), wobei wir uns in unseren Ausführungen auf die abstrakte, schematische $[\mathrm{ADJ}+\mathrm{PP}]_{\text {kausal }}$ beschränkt haben. Als Form-Bedeutungs-Paare sind die einzelnen Instanziierungen dieser Konstruktion durch Vererbungsbeziehungen miteinander verbunden. Folgende Vererbungsbeziehungen konnten unterschieden werden: (a) die Beispiel-von-Relation zwischen einer prototypischen Konstruktion wie (33) fr. Il est triste de la perte de sa mère (,Er ist traurig über den Verlust seiner Mutter') und dem Beleg (11) fr. Elle était rouge de colère. (,Sie war rot vor Wut $^{`}$ ); b) die metonymische Beziehung, nämlich Farbe des Gesichts für physischen Zustand, bei einer idiomatischen Interpretation des Beispiels (20) fr. La jeune fille était devenue rouge de honte, (,Die junge Frau war rot vor Scham geworden'); und c) die metaphorische Beziehung wie bei Beispiel (34) it. Sono nero dalla rabbia oggi, (,Ich bin schwarz vor Wut heute', d. h. ,sehr wütend'). Außerdem liegt eine Teil-Ganzes-Beziehung zwischen den Konstruktionen (34) it. Sono nero dalla rabbia oggi (,Ich bin schwarz vor Wut heute', d. h. ,sehr wütend') und (35) Sono nero oggi, (,Ich bin schwarz heute', d. h. ,sehr sauer/ wütend/schlecht drauf heute'). Weiter konnte festgestellt werden, dass die Konstruktion [ADJ+PP] kausal mit einer anderen, teilschematischen Konstruktion durch metaphorische Links verbunden ist und zwar mit der PhK [X KOPULA ADJ ${ }_{\text {extr. Zustand+Präp }}$ dt. vor/ fr.de/ it.di $\left.+\mathrm{Y}_{\text {Zustand/Gefüh//Bedürfnis }}\right]_{\text {kausal } \pm \text {, }}$ die eher einen extremen Zustand zum Ausdruck bringt, wobei ihre Kausalkomponente nicht völlig verblasst ist.

(ii) Wie lassen sich die wörtlichen Kausalkonstruktionen von den phraseologischen unterscheiden?

Die von uns diskutierten kausalen Konstruktionen mit einem Adjektiv und die $\mathrm{PhK}$ sind durch verschiedene Kompositionalitäts- und Lexikalisierungsgrade gekennzeichnet. Die Bedeutung der nicht-lexikalisierten Kausalkonstruktionen ist kompositioneller Natur, die kausale Ge- 
samtbedeutung der Konstruktion ist aufgrund der Bedeutung der konstituierenden Bestandteile zu rekonstruieren. Man denke hier etwa an ein Beispiel wie dt. Seine Nase ist schwarz vom Tabak oder Ich bin sehr müde von der Reise. Je mehr eine Instanziierung lexikalisiert ist, desto weniger ist die Bedeutung auf kompositionelle Art zu ermitteln. Als Illustrierung erinnern wir an das oben besprochene italienische Beispiel (35) Sono nero oggi (,Ich bin schwarz heute', d. h. ,sehr sauer/wütend/schlecht drauf heute').

Parallel dazu kann eine gewisse Relation zwischen Lexikalisierungsgrad/Idiomatizität und Kausalität festgestellt werden: Je idiomatischer ein Beispiel, desto weniger drückt es eine kausale Bedeutung aus oder sie ist in der Wendung zwar präsent, jedoch nicht mehr vordergründig. Dies wurde deutlich bei der Besprechung des Beispiels rot vor Wut, das in seiner wörtlichen Lesart eine kausale Bedeutung vermittelt (d. h. tatsächlich rotes Gesicht verursacht durch die starke Emotion der Wut). Als fester, fixierter, lexikalisierter Ausdruck bedeutet rot vor Wut einen extremen Zustand der Wut. Diese Dualität oder gegenseitige Beziehung lässt sich wie folgt darstellen:

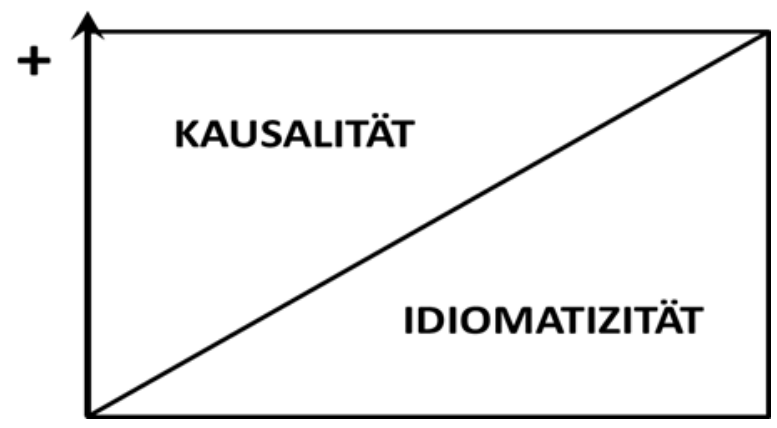

Abbildung 1: die Dualität zwischen Kausalität und Idiomatizität

Zusammenfassend lässt sich sagen: Je mehr Kausalität, desto weniger Idiomatizität, oder umgekehrt, je idiomatischer die Instanziierung einer Konstruktion, desto weniger drückt sie eine kausale Bedeutung aus.

(iii) Wie lässt sich die Grenze zwischen einer wörtlichen Konstruktion, einem Phraseologismus und einer so genannten Phrasem-Konstruktion definieren?

Wie wir festgestellt haben, sind häufig die Grenzen zwischen den einzelnen Instanziierungen einer Konstruktion einerseits und zwischen freiem und fixiertem Gebrauch andererseits zum Teil so minimal, dass sie manchmal schwer zu erfassen sind (rot vor Wut kann z. B. sowohl wörtlich als auch idiomatisch interpretiert werden). Ausgehend von der Kompositionalität einer Wendung und ihrem Lexikalisierungsgrad plädieren wir im Folgenden für ein Kontinuum zwischen den verschiedenen Instanziierungen. Während an einem Pol dieses Kontinuums freie, nicht-lexikalisierte Instanziierungen der Konstruktion $[\mathrm{ADJ}+\mathrm{PP}]_{\text {kausal }}$ vorzufinden sind, finden wir am anderen Pol die idiomatischen Wendungen. In der Mitte sind die analysierten PhK, da sie im Grenzbereich zwischen Syntax und Phraseologie liegen und sowohl lexikalisch feste Positionen als auch freie Slots enthalten. 
KONTINUUM

$[\mathrm{ADJ}+\mathrm{PP}]$

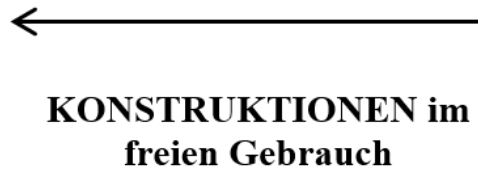

Peter ist rot vor Wut. (wörtlich)

\section{PHRASEM- KONSTRUKTIONEN}

Peter ist verrückt vor Eifersucht.

\section{PHRASEOLOGISMEN/ IDIOME}

Peter ist rot vor Wut. (d. h. ,sehr wütend')

Abbildung 2: Kontinuum zwischen wörtlichen Konstruktionen, Phrasem-Konstruktionen und Phraseologismen/Idiomen (anhand des Deutschen)

(iv) Inwiefern bietet die Konstruktionsgrammatik ein effizientes Modell zur Analyse und Beschreibung phraseologischer Konstruktionen?

In der Konstruktionsgrammatik werden phraseologische bzw. idiomatische Wendungen - im Gegensatz zu anderen Modellen - nicht als periphere sprachliche Erscheinungen betrachtet (Fillmore et al. 1999). Benigni et al. (2015: 4f.) schreiben diesbezüglich:

$\mathrm{CxG}$ arose precisely from the need to find a theoretical model that was also adequate to describe phraseological-type units (Croft and Cruse 2004: 225) whose form is difficult to predict (such as collocations) as well as its content (such as idiomatic expressions) and which present many descriptive difficulties for different grammatical models, thus leading them to be viewed as marginal phenomena to be relegated to the lexicon. In $\mathrm{CxG}$, phraseologisms, thanks in part to the frequency with which they are recognized within the linguistic system, acquire importance and interest.

Die Tatsache, dass die $[\mathrm{ADJ}+\mathrm{PP}]_{\text {kausal-Konstruktion sowohl im freien als auch im fixierten }}$ Gebrauch vorkommt und dass die Grenzen zwischen den unterschiedlichen Instanziierungen fließend sind, ist ein Beweis dafür, dass der Bereich der Grammatik von dem des Lexikons nicht scharf zu trennen ist. Aus unseren Ausführungen ist außerdem deutlich geworden, dass die Konstruktionsgrammatik und die Phraseologie das gemeinsame Interesse für die idiosynkratischen, nicht-prädiktablen und nicht-kompositionellen Erscheinungen im Sprachsystem teilen, sie divergieren jedoch in der Terminologie, in der Perspektive und in ihren Zielen (cf. Gries 2008: 10). Wie die Phraseologie sieht die Konstruktionsgrammatik eine holistische Analyse (s. Croft 2001) der beschriebenen Konstruktion vor. Während jedoch die Phraseologie ihr Interesse an den phraseologischen Einheiten begrenzt, strebt die Konstruktionsgrammatik ein komplettes Inventar der Konstruktionen einer Sprache an und dabei untersucht sie die Vererbungsbeziehungen zwischen ihnen (cf. u. a. Goldberg 1995). Wir stimmen mit Gries (2008: 10) überein, wenn er schreibt:

From this, it of course also follows in turn that phraseological research has a lot to offer to these theories in terms of descriptive work as well as exploration of the ontological status of phraseological elements. In the opposite direction, phraseological research can benefit from the elaborate theoretical apparatus and the cognitively plausible background provided by Cognitive Grammar and Construction Grammar.

Wir sind der Meinung, dass einige Grundannahmen und Prinzipien der Konstruktionsgrammatik (wie die Annahme abstrakter und schematischer Konstruktionen und die Konzeptualisierung des Lexikon-Syntax-Kontinuums) der Phraseologie sehr nützlich sein können, um Phraseologismen im Sprachsystem einheitlicher zu definieren. Die Konstruktionsgrammatik ist ein integratives Modell, das es ermöglicht, die Beispiele-Vielfalt unabhängig von ihrem 
Lexikalisierungsgrad zu erfassen. Aufgrund der engen Form-Bedeutungsrelation können die unterschiedlichsten Realisierungen derselben instanziierten Konstruktion [ADJ+PP] kausal adäquater beschrieben werden. Außerdem ist festzustellen, dass der konstruktionsgrammatische Ansatz eine einheitliche Untersuchung der Kausalkonstruktion ermöglicht, da die in diesem Beitrag analysierte Realisierungsvielfalt nicht wie eine willkürliche, unstrukturierte Beispielliste beschrieben wird, sondern vielmehr als ein Netzwerk von Konstruktionen und Instanziierungen, die durch metaphorische, metonymische, bzw. weitere Vererbungsbeziehungen nicht nur miteinander, sondern auch mit der PhK [X KOPULA ADJ $J_{\text {extr. Zustand }+ \text { Präp }}$ dt. $_{\text {. }}$

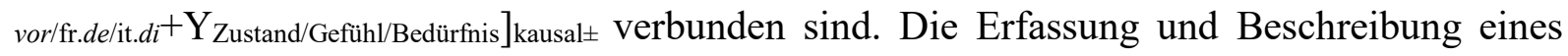
solchen Netzwerks bildet die erste Etappe zur Konstitution eines Konstruktikons.

(v) Welche Erkenntnisse konnten aus der kontrastiven Analyse (Deutsch-FranzösischItalienisch) solcher Konstruktionen gewonnen werden?

Wie wir gezeigt haben, lassen sich die Instanziierungen der untersuchten Kausalkonstruktion in sehr ähnlicher Form, auch phraseologisch mit der Realisierung desselben Adjektivs, in den untersuchten Sprachen wiederfinden. Dies ist dadurch zu erklären, dass die Konstruktion $[\mathrm{ADJ}+\mathrm{PP}]_{\text {kausal }}$ in vielen Fällen metonymisch motiviert ist; daher wundert es nicht, dass unterschiedliche Sprachen auf ähnliche bzw. identische Mittel zurückgreifen, um Kausalität zu versprachlichen. Jedoch wurden auch Divergenzen gefunden. Während z. B. das Deutsche und das Italienische jeweils eine Präferenz für die Farbe Gelb bzw. Grün in Assoziierung mit Neid und Eifersucht verbinden, kommt im Französischen fast ausschließlich die Farbe Grün mit denselben Emotionen in Frage. Außerdem liegt nur im Italienischen und im Deutschen eine phraseologische Instanziierung der Kausalkonstruktion vor, in der die Farbe Schwarz metaphorisch für schlechte Laune bzw. das Wütendsein steht.

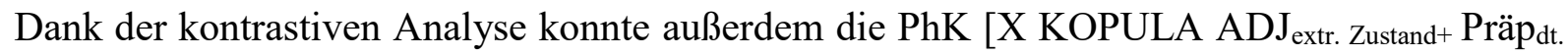

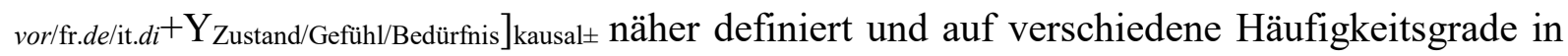
den drei untersuchten Sprachen hingewiesen werden. Außerdem wurde gezeigt, dass das Deutsche bei der Präpositionsauswahl - anders als das Französische und Italienische - differenzierter vorgeht. Aus diesen Gründen erscheint uns eine kontrastive Analyse von Konstruktionen (sowohl im freien als auch im fixierten Gebrauch) als sehr nützlich, um die kognitiven Prozesse zu verstehen, die den unterschiedlichen Sprachen unterliegen. Wir sind uns dessen bewusst, dass die in unserem Beitrag präsentierte kontrastive Analyse nicht umfangreich genug ist und dass dieses Thema einer weiteren, quantitativen Untersuchung bedarf. Dies stellt ein Forschungsdesiderat dar, das Thema zukünftiger Studien sein könnte.

\section{Korpora}

DeReKo, http://www.dwds.de. [10.12.2016]

SketchEngine, http://the.SketchEngine.co.uk. [10.12.2016]

\section{Literatur}

Ballestracci, Sabrina (2011): „Die kausalen Verknüpfungsmittel des Deutschen und des Italienischen. Eine kontrastive Beschreibung unter formalem und funktionalem Aspekt". Linguistik online 49: 75-89. 
Benigni, Valentina et al. (2015): "How to Apply CxG to Phraseology: A Multilingual Research Project". Journal of Social Sciences 11/3, special issue on Phraseology, Phraseodidactics and Construction Grammar(s). http://thescipub.com/PDF/ofsp.10003.pdf.

Berlin, Brent/Kay, Paul (1969): Basic Color Terms: Their Universality and Evolution. Berkeley: University of California Press.

Burger, Harald (2007): Phraseologie - Eine Einführung am Beispiel des Deutschen. Berlin: Erich Schmidt.

Croft, William A. (2001): Radical Construction Grammar. Syntactic Theory in Typological Perspective. Oxford: Oxford University.

Croft, William/Cruse, Alan D. (2004): Cognitive Linguistics. Cambridge: Cambridge University.

De Knop, Sabine (2013): „Eine Beschreibung von Konstruktionen mit Farbbezeichnungen im Rahmen der Konstruktionsgrammatik“. In: De Knop, Sabine/Mollica, Fabio/Kuhn, Julia (eds): Konstruktionsgrammatik und romanische Sprachen. Frankfurt a. M., Peter Lang: 111-136.

De Knop, Sabine (2015): "Causal constructions with an adjective in German and French: typological and pedagogical considerations”. Journal of Social Sciences 11/3, special issue on Phraseology, Phraseodidactics and Construction Grammar(s): 289-303. http://thescipub.com/abstract/10.3844/ofsp.10085.

Derrig, Sandra (1978): "Metaphor in the Color Lexicon". In: Farkas, Donka/Jacobsen, Wesley M./Todrys, Karol (eds.): Papers from the Parasession on the Lexicon. Chicago, Chicago Linguistic Society: 85-96.

Dirven, René (1995): “The construal of cause: The case of cause prepositions". In: Taylor, John/MacLaury, Robert (eds.): Language and the Cognitive Construal of the World. Berlin, Mouton de Gruyter: 95-118.

Dirven, René (1997): "Emotions as cause and the cause of emotions". In: Niemeier, Susanne/Dirven, René (eds.): The Language of Emotions: Conceptualization, Expression, and Theoretical Foundation. Amsterdam, Benjamins: 55-83.

Dobrovol'skij, Dmitrij/Piirainen, Elisabeth (2002): Symbole in Sprache und Kultur. Studien zur Phraseologie aus kultursemiotischer Perspektive. Bochum: Brockmeyer.

Dobrovol'skij, Dmitrij (2011): „Phraseologie und Konstruktionsgrammatik“. In: Lasch, Alexander/Ziem, Alexander (eds.): Konstruktionsgrammatik III. Aktuelle Fragen und Lösungsansätze. Tübingen, Stauffenburg: 110-130.

Donalies, Elke (2009): Basiswissen Deutsche Phraseologie. Tübingen: Narr.

Eisenberg, Peter (2004): Grundriß der deutschen Grammatik. Bd. 2: Der Satz. 2. überarbeitete und aktualisierte Aufl. Stuttgart: Metzler.

Fillmore, Charles/Kay, Paul/O'Connor, Mary (1988): “Regularity and Idiomacity in Grammatical Constructions: The Case of Let Alone”. Language 64/3: 501-538.

Fleischer, Wolfgang (1997): Phraseologie der deutschen Gegenwartssprache. Tübingen: Niemeyer.

Frei, Henri (1939) : «Sylvie est jolie des yeux ». In : Bally, Charles (ed.) : Mélanges de Linguistique offerts à Charles Bally. Genève, Georg : 187-192.

Girdeniene, Skaidra (2006): „Zum Ausdruck der inneren Ursache durch Präpositionalphrasen im Deutschen und Litauischen“. Kalbotyra 56/3: 48-55. http://archive.minfolit. 1t/arch/15001/15441.pdf. 
Goldberg, Adele (1995): Constructions. A Construction Grammar Approach to Argument Structure. Chicago: University of Chicago.

Goldberg, Adele (2006): Constructions at Work: The Nature of Generalization in Language. Oxford: Oxford University Press.

Goossens, Louis (1990): "Metaphtonymy: the interaction of metaphor and metonymy in expressions for linguistic action". Cognitive Linguistics 1/3: 323-340.

Grevisse, Maurice (1936): Le Bon Usage. Cours de Grammaire Française et de Langage Français. Gembloux: Duculot.

Gries, Stefan (2008): "Phraseology and linguistic theory: A brief survey". In: Granger, Sylviane/Meunier, Fanny (eds.): Phraseology: An interdisciplinary perspective. Amsterdam/Philadelphia, Benjamins: 3-25.

Hinrichs, Uwe (2004): „Vorbemerkungen zum analytischen Sprachtyp in Europa“. In: Hinrichs, Uwe/Büttner, Uwe (eds.): Die europäischen Sprachen auf dem Wege zum analytischen Sprachtyp. Wiesbaden, Harrassowitz: 169-196.

Khoo, Christopher/Chan, Syin/Niu, Yun (2002): "The many facets of the cause-effect relation". In: Green, Rebecca/Bean, Carol /Myaeng, Sung Hyon (eds.): The Semantics of Relationships: An Interdisciplinary Perspective. Dordrecht, Kluwer: 51-70.

Kövecses, Zoltàn (2000): Metaphor and Emotion. Language, Culture, and Body in Human Feeling. Cambridge: Cambridge University.

Lakoff, George/Johnson, Mark (1980): Metaphors We Live By. Chicago: Chicago University.

Laptieva, Ekaterina (2017): „rot vor Wut vs. rot vor Blut: eine korpusbasierte Untersuchung kausaler vor-Phrasen in Kopulasätzen“. Deutsche Sprache 4: 335-361.

Léard, Jean-Marcel/Marengo, Sébastien (2005) : "Pour une typologie des compléments adjectivaux: Arguments, quasi-arguments et non-arguments ». In : Jacques, Francois (ed.) : L'adjectif en Français et à Travers les Langues. Actes du colloque international de Caen (28-30 juin 2001). Caen, Presses universitaires de Caen : 387-402.

Lohde, Michael (2006): Wortbildung des Modernen Deutschen. Ein Lehr- und Übungsbuch. Tübingen: Narr.

Niemeier, Susanne (1998): "Colourless green ideas metonymize furiously". Rostocker Beiträge zur Sprachwissenschaft 5: 119-146.

Primus, Beatrice (1997): „Der Wortgruppenaufbau in der Geschichte des Deutschen: Zur Präzisierung von synthetisch vs. analytisch“. Sprachwissenschaft 22: 133-159.

Radden, Günter/Dirven, René (2008): Cognitive English Grammar. Amsterdam/Philadelphia: Benjamins.

Rostila, Jouni (2011): „Phraseologie und Konstruktionsgrammatik. Konstruktionsansätze zu präpositionalen Funktionsverbgefügen“. In: Prinz, Michael/Richter-Vapaatalo, Ulrike (eds.): Idiome, Konstruktionen, , verblümte Rede'. Beiträge zur Geschichte der germanistischen Phraseologieforschung. Stuttgart, Hirzel: 263-282.

Salles, Mathilde (1998) : «La construction converse 'Etre un peu lent de la tête mais rapide des pieds' ». La Linguistique 34/1 : 121-136.

Siemund, Peter (2004): „Analytische und synthetische Tendenzen in der Entwicklung des Englischen“. In Hinrichs, Uwe/Büttner, Uwe (eds.): Die europäischen Sprachen auf dem Wege zum analytischen Sprachtyp. Wiesbaden, Harrassowitz: 169-196.

Smirnova, Elena/Mortelmans, Tanja (2010): Funktionale Grammatik. Berlin: de Gruyter. 
Soriano, Cristina/Valenzuela, Javier (2009): "Emotion and colour across languages: Implicit associations in Spanish colour terms". Social Science Information 48/3: 421-445.

Staffeldt, Sven (2011): „Wann rot und wann schwarz gesehen wird. Bemerkungen zur Methode der Analyse phraseologischer Bedeutungen anhand zweier Farbphraseologismen“. In: Groh, Arnold (ed.): Was ist Farbe? Bunte Beiträge aus der Wissenschaft. Berlin, Weidler: 123-143.

Sweetser, Eve (1990): From Etymology to Pragmatics: Metaphorical and Cultural Aspects of Semantic Structure. Cambridge: Cambridge University.

Wierzbicka, Anna (1990): "The meaning of color terms: Semantics, culture and cognition". In: Cognitive Linguistics 1: 99-150.

Wulff, Stefanie (2012): "Idiomaticity". In: Robinson, Peter (ed.): The Routledge Encyclopedia of Second Language Acquisition. London, Routledge: 291-293.

Zeschel, Arne (2012): Incipient Productivity. A Construction-based Approach to Linguistic Creativity. Berlin/Boston: de Gruyter.

Ziem, Alexander/Alexander Lasch (2013): Konstruktionsgrammatik: Konzepte und Grundlagen Gebrauchsbasierter Ansätze. Berlin: de Gruyter.

Ziem, Alexander (2014/im Druck): ,(Konzeptuelle) Metaphern in der Kognitiven Konstruktionsgrammatik“. In: Köpcke, Klaus-Michael/Spieß, Constanze (eds.): Metapher und Metonymie. Theoretische, methodische und empirische Zugänge. Berlin/Boston, de Gruyter: 51-79.

Zimmer, Dieter (1996): Redensarten: Über Trends und Tollheiten im neudeutschen Sprachgebrauch. München: Wilhelm Heyne. 